\title{
Nonlinear Heat Radiation Induces Thermal Rectifier in Asymmetric Holey Composites
}

\author{
Weiwei Zhu', Gaomin Wu', Hong Chen and Jie Ren* \\ Center for Phononics and Thermal Energy Science, China-EU Joint Center for Nanophononics, Shanghai Key Laboratory \\ of Special Artificial Microstructure Materials and Technology, School of Physics Science and Engineering, Tongji University, \\ Shanghai, China
}

Asymmetric design and nonlinearity are essential to give rise to thermal rectification. In addition to the linear Fourier's law of thermal conduction, we introduce the nonlinear thermal radiation following Stefan-Boltzmann law to asymmetric holey composites to realize the thermal rectifier. The thermal rectification results from the competition between the linear thermal conduction and the nonlinear thermal radiation, which we explain as a thermal resistor network with the linear and nonlinear components connected in

OPEN ACCESS

Edited by:

Nuo Yang,

Huazhong University of Science and Technology, China

Reviewed by:

Qiang Cheng,

Huazhong University of Science and Technology, China

Dahai He,

Xiamen University, China

*Correspondence:

Jie Ren

xonics@tongji.edu.cn

tThese authors have contributed equally to this work.

Specialty section: This article was submitted to Nanoenergy Technologies and Materials,

a section of the journal Frontiers in Energy Research

Received: 22 December 2017 Accepted: 14 February 2018 Published: 05 March 2018

Citation:

Zhu W, Wu G, Chen H and Ren J (2018) Nonlinear Heat Radiation

Induces Thermal Rectifier in Asymmetric Holey Composites.

Front. Energy Res. 6:9. doi: 10.3389/fenrg.2018.00009 series-parallel. All the results are confirmed by an analytical model that is composed of one central nonlinear thermal radiation region sandwiched by two linear conduction regions with different conductances. We can get the optimal rectification effect around the room temperature when the temperature difference is fixed, which allows the application of the designed materials to energy-saving buildings.

Keywords: thermal rectification, thermal radiation, non-reciprocal heat transfer, metamaterial, heat conduction, thermal diode, energy conservation

\section{INTRODUCTION}

In analogy to the familiar electric diode in modern electronics, thermal rectifier is a device that can achieve non-reciprocal heat transport. Theoretical models for thermal rectifiers are proposed relying on various mechanisms (Roberts and Walker, 2011), including different temperature-dependent thermal properties between dissimilar materials at a contact (Stevenson et al., 1991; Dames, 2009; Kobayashi et al., 2009), asymmetric design of nanostructure (Wu and Li, 2008; Yang et al., 2009), and quantum thermal systems, such as quantum dots and other quantum heterojunctions (Scheibner et al., 2007; Ruokola et al., 2009; Wu and Segal, 2009; Ren and Zhu, 2013a). Similar concepts are even extended to the spin Seebeck diode and transistor (Ren, 2013; Ren and Zhu, 2013b; Ren et al., 2013), and the multiferroic thermal diode (Chotorlishvili et al., 2015), multiferroic switch and memory (Li et al., 2014). All these device concepts lay the foundation for the emerging field: phononics, where scientists are devoted to control heat flow and thermal information carried by phonons and beyond with electronic analogs (Li et al., 2012; Maldovan, 2013; Ren and Li, 2015).

It has been well established that asymmetric design and nonlinearity are essential to give rise to thermal rectification (Roberts and Walker, 2011; Li et al., 2012). Thus, there have been more theoretical studies of thermal rectification focusing on nonlinear systems (Wehmeyer et al., 2017). Nonlinearity can be provided in different ways to design a thermal rectifier, including one-dimensional nonlinear lattice (Terraneo et al., 2002; Li et al., 2004; Hu and Yang, 2005), nonlinear electron gas dispersion in conductors (Segal, 2008), nonlinear Schrödinger equation (Li and Ren, 2014), nonlinear near-field radiation (Otey et al., 2010; Basu and Francoeur, 2011), and even a vapor generation rectifier (Ding et al., 2017). 
Punching holes in an isotropic material can induce a new heat transfer mechanism-radiation or convection (if the hole is filled with fluid or gas) in addition to the thermal conduction of the material. Thus, some interesting thermal work of materials with holes has been studied for thermal buckling analysis (Zhao et al., 2009), thermal rectifier (Hu et al., 2017) and thermal cloaking (Maldovan, 2013). Yet, few researches of materials with holes consider the heat radiation process on the surface of holes as a nonlinear procedure required in thermal rectification.

In this paper, we design a simple model of thermal rectifier, in which required nonlinearity is met by thermal radiation which is proportional to the fourth power of temperature within the framework of Stefan-Boltzmann Law. The rectification design comes from the asymmetry of the structure and the competition between nonlinear thermal radiation and linear thermal conduction.

We discuss how asymmetry and nonlinearity works to achieve thermal rectification in our model, with results of simulation and analytical explanation. In our simulation models, we consider two kinds of heat transfer mechanisms: (i) thermal conduction that is linear as we consider constant thermal conductivity and (ii) thermal radiation that is proportional to the fourth power of temperature. We report the simulation results of our holey diodes that have non-reciprocal heat transfer due to the nonlinearity of the thermal radiation and the asymmetric geometry. We obtain the clear thermal rectification, and find that there is a best average temperature, where the rectification reaches maximum, when the temperature difference is fixed. All the simulation results can be analytically explained by a simple thermal circuit model, which is composed of a central thermal radiation region sandwiched by two conduction regions with different thermal conductances.

\section{RESULTS}

Since there are already thermal diodes achieved with different temperature-dependent conductivity (Dames, 2009) or emissivity (Roberts and Walker, 2011), in our study we consider the bulk material itself to be homogenous and isotropic. In the homogenous material, the local heat flux density is expressed as $\vec{J}=-\kappa \nabla T$, according to Fourier's Law, where $J$ is the heat flux, $\kappa$ is the material's thermal conductivity and on the vacuum interface of the holes, heat transfer follows the Stefan-Boltzmann Law $\vec{J}=-\varepsilon \sigma \nabla T^{4}$, where $\varepsilon$ is the emissivity and $\sigma$ is the StefanBoltzmann constant. For simplification, we consider no medium inside the holes so that there is no need to consider the convection and conduction mechanism of the medium. It has been proven that (Li and Ren, 2014) combination of nonlinearity and asymmetry is an effective method to give rise to rectification. It is, therefore, reasonable to infer that holey structures could be able to transfer heat non-reciprocally and the analytical explanation will be demonstrated later.

\subsection{Simulation Results for a Feasible Holey Diode Model}

To achieve heat rectifier effect, we design a structure with dense periodic organization of circle holes in a pattern, only on a half of the structure itself, as shown in Figure 1A,B. This system is too complicated to be tackled analytically. Therefore, we perform two dimensional finite-element simulations, to investigate the heat transport across the structure. The temperature of the left and the right boundaries of the diode are fixed at $T_{0}+\Delta T$ and $T_{0}-\Delta T$, respectively, where $T_{0}$ represents the average temperature of the structure approximately and $2 \Delta T$ the temperature difference of two terminals. In the reverse temperature biased scenario $(\Delta T=-\Delta T)$, the temperatures of the boundaries are exchanged.

If the magnitudes of thermal energy transfer in the two directions are different, we can safely say that there is a thermal rectification. Since no energy escapes from the system in the twodimension and infinite periodic structure we proposed, we can calculate the total heat flux passing through the whole structure by integrating the normal heat flux along the boundary either the left one or the right one. We define $J_{+}$as heat flux flowing from left to right when the value of $\Delta T$ is positive in the setting of the boundary temperature, while $J_{-}$with negative $\Delta T$ denotes the right-to-left heat flux in the reversed temperature biased scenario. To quantify the magnitude of thermal rectification, we define a rectification factor $R=\frac{J_{+}-J_{-}}{J_{-}}$.

Heat flux in the forward and reverse bias as well as the corresponding rectification factor as a function of $\Delta T$ are plotted in Figure 1C. As shown in the plot, the heat transfer in the forward bias is always stronger than in the reverse bias. $J_{+}, J_{-}$, and rectification factor $R$ are shown to increase with the increasing $\Delta T$, in an approximately linear relationship. Moreover, it is shown in Figure $1 \mathrm{D}$ that although $J_{+}$and $J_{-}$increase with the average temperature $T_{0}, R$ does not monotonically increase with $T_{0}$, and reaches its maximum at about $330 \mathrm{~K}$ in this settings.

The thermal rectification is achieved by satisfying the required asymmetry and nonlinearity. Therefore, if the holes in our proposed model are punched exactly in the center of the structure, rectification would not appear, since the geometry of the structure is totally symmetric. To achieve a strong rectification effect, we design the model in an asymmetric way. The asymmetric setup is constructed by using two materials with different constant thermal conductivity, where material on the left holey side has thermal conductivity $\kappa_{L}$ and the right complete side $\kappa_{R}$. Both the results of the simulation and the sketch of the new model are shown in Figure 2. Note the left and right materials can be identical so that $\kappa_{L}=\kappa_{R}$ is allowed.

As shown in Figure 2C, we keep one of the $\kappa_{L}$ and $\kappa_{R}$ in the new model equal to the thermal conductivity of the single material model, $1.7 \mathrm{~W} /(\mathrm{m} \cdot \mathrm{K})$, and compare the new model with the previous one. The simulation results show that if $\kappa_{L}=1.7 \mathrm{~W} /(\mathrm{m} \cdot \mathrm{K})$ and $\kappa_{R}=1$ or $0.7 \mathrm{~W} /(\mathrm{m} \cdot \mathrm{K})$, the rectification factor is improved, since not only the geometry but also the thermal properties are asymmetric in this model. However, if $\kappa_{R}=1.7 \mathrm{~W} /(\mathrm{m} \cdot \mathrm{K})$ and $\kappa_{L}=10$ or $5 \mathrm{~W} /(\mathrm{m} \cdot \mathrm{K})$, even though with stronger asymmetry, the rectification effect becomes weaker.

To understand the phenomenon in a more clear way, we introduce the equivalent thermal circuit of the model in Figure 2B. $G_{R}$ is the conductance of the right half of the structure, which can be easily calculated by $G_{R}=\kappa_{R} / 0.417 \mathrm{~m}$, since the right half is totally homogenous. However, the left half is more complicated. $G_{L}$ is the equivalent conductance of the conduction mechanisms 

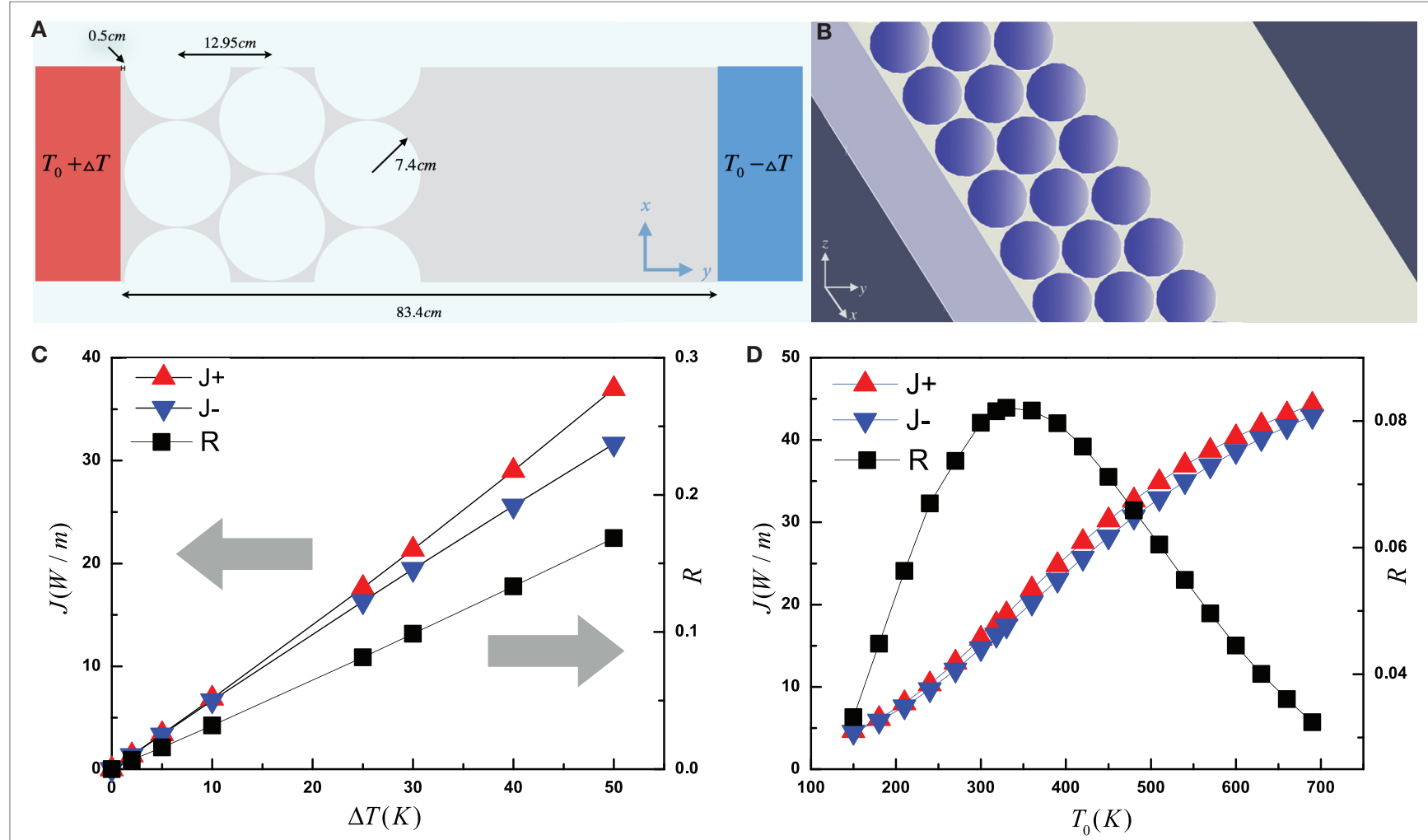

FIGURE 1 | Simulation model of holey concrete structure. (A) Sketch of the thermal diode model with periodic boundary condition in the vertical direction. The width of the structure is $83.4 \mathrm{~cm}$. For the hole patterns, the hole diameter is $7.4 \mathrm{~cm}$, and the horizontal distance between two centers of the adjacent holes is $12.95 \mathrm{~cm}$. The gray area is the body of the concrete structure, and the blue area is the background that we consider as vacuum. The thermal conductivity of the concrete is $\kappa=1.7 \mathrm{~W} /(\mathrm{m} \cdot \mathrm{K})$ and emissivity is set as $\varepsilon=1$. (B) Schematic graph depicting the infinite periodic holey thermal diode. We consider the model infinite in $x$ and $z$ axis direction. (C) Heat flux in forward $\left(J_{+}\right)$and reverse $\left(J_{-}\right)$temperature biased system and rectification factor $R$ versus $\Delta T$ for thermal rectifier model in $(A)$ and $(B)$. $T_{0}$ is fixed at $318 \mathrm{~K}$. (D) Heat flux in forward and reverse temperature biased system and rectification factor versus $T_{0}$. $\Delta T$ is fixed at $25 \mathrm{~K}$.

on the left. Moreover, $G_{r a d}$ denotes the equivalent conductance contributed by the radiation of holes on the left. Due to the circle geometry of the holes, $G_{L}$ can not be easily expressed like $G_{R}$. But, It is evident that $G_{L} \propto \kappa_{L}$ and $G_{r a d}$ has nonlinear positive relationship with temperature. Therefore, when $\kappa_{L}$ is larger, so does $G_{L}$, the resistance of conduction would be smaller guiding more heat flux flow through it rather than through radiation. Since little flux through radiation means little nonlinearity, although in this case the model has stronger asymmetry, the nonlinearity is weaker leading to the weaker diode effect. In the proposed model, thermal resistance of conduction and radiation mechanisms is in series and in parallel. In the holey side, heat can transfer in two channels, and competition between the channels weaken the required nonlinearity to give rise to rectification. To improve the rectification effects, we construct a more effective model, where thermal resistances of radiation and conduction are only in series, in the next subsection. This case also renders us an analytical explanation.

\subsection{Two Infinite Plate Model with Analytical Explanation}

As shown in the Figure 3, we analyze the heat transfer between two infinite parallel plates of different conductance in the simplified model. The thermal conductance instead of thermal conductivity are considered here, and can be calculated through $G_{i}=\kappa_{i} / w_{i}$, where $\kappa_{i}$ is the thermal conductivity and $w_{i}$ represents the thickness of two infinite plates, with $i=1,2$. In this case, the combined processes of thermal conduction and thermal radiation form an equivalent thermal circuit in series. Since all the heat flux leaving one plate will arrive at the other plate, the heat flux through the three regions (I, II, III) would be the same:

$$
J=G_{1}\left(T_{0}+\Delta T-T_{1}\right)=\varepsilon \sigma\left(T_{1}^{4}-T_{2}^{4}\right)=G_{2}\left(T_{2}-T_{0}+\Delta T\right) .
$$

Note the Stefan-Boltzmann constant $\sigma$ is at -8 order of magnitude in SI units, which makes the nonlinear radiation contribution rather weak. If we consider the situation where $\Delta T$ as well as $J$ is small, we can obtain the expressions of $J_{+}$and $J_{-}$up to the second order of thermal bias, as following:

$$
\begin{aligned}
J_{ \pm} \approx & \frac{4 \varepsilon \sigma G_{1} G_{2} T_{0}^{3}}{G_{1} G_{2}+4 \varepsilon \sigma T_{0}^{3}\left(G_{1}+G_{2}\right)} 2 \Delta T \\
& \pm \frac{24(\varepsilon \sigma)^{2} G_{1} G_{2}\left(G_{1}-G_{2}\right)}{\left[G_{1} G_{2} / T_{0}^{3}+4 \varepsilon \sigma\left(G_{1}+G_{2}\right)\right]^{2}} \frac{(2 \Delta T)^{2}}{T_{0}} .
\end{aligned}
$$

The first term of equation (2) is a linear contribution that has a clear physical picture: with linear response, region II is 

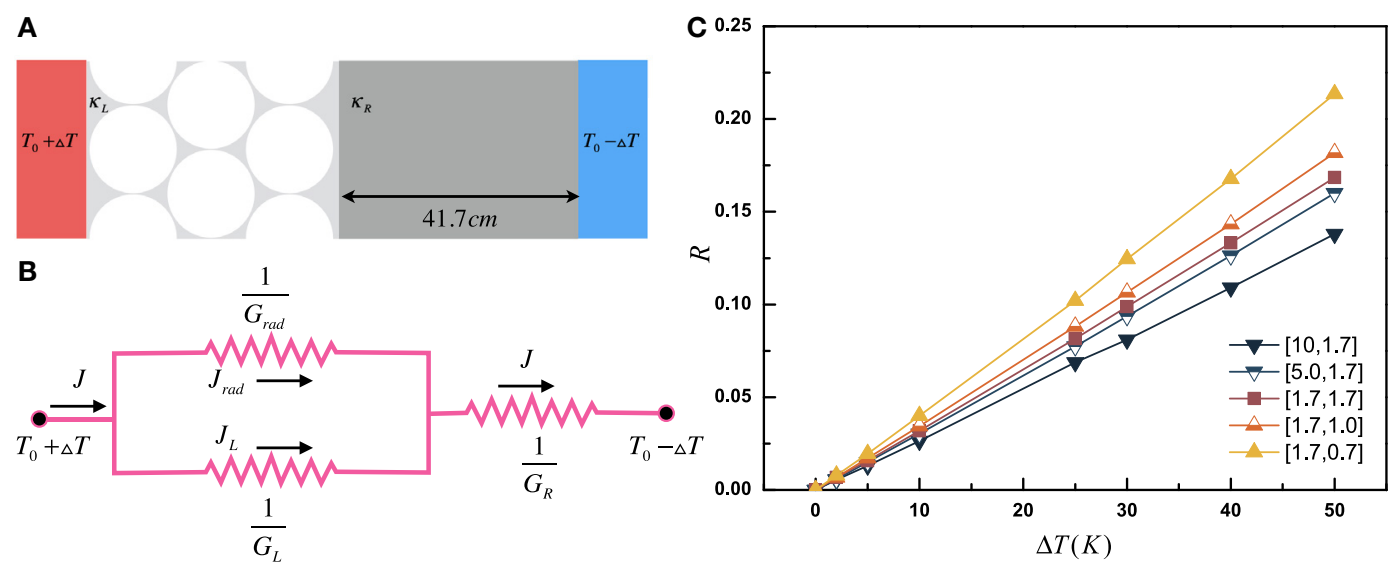

FIGURE 2 | (A) Sketch of the two-material junction model, which have different thermal conductivity in left half $\left(\kappa_{L}\right)$ and the right half $\left(\kappa_{R}\right)$. Parameters and geometries are the same as the previous ones. (B) Schematic graph depicting the equivalent thermal circuits of the new model. Note that thermal resistance $R=1 / G$, where $G$ is the corresponding thermal conductance. (C) Thermal rectification factor $R$ versus $\Delta T$ of the model with different combination of thermal conductivity of left and right with $\left[\kappa_{L}, \kappa_{R}\right]$ in the legend. Unit of all thermal conductivity mentioned is $\mathrm{W} /(\mathrm{m} \cdot \mathrm{K})$.
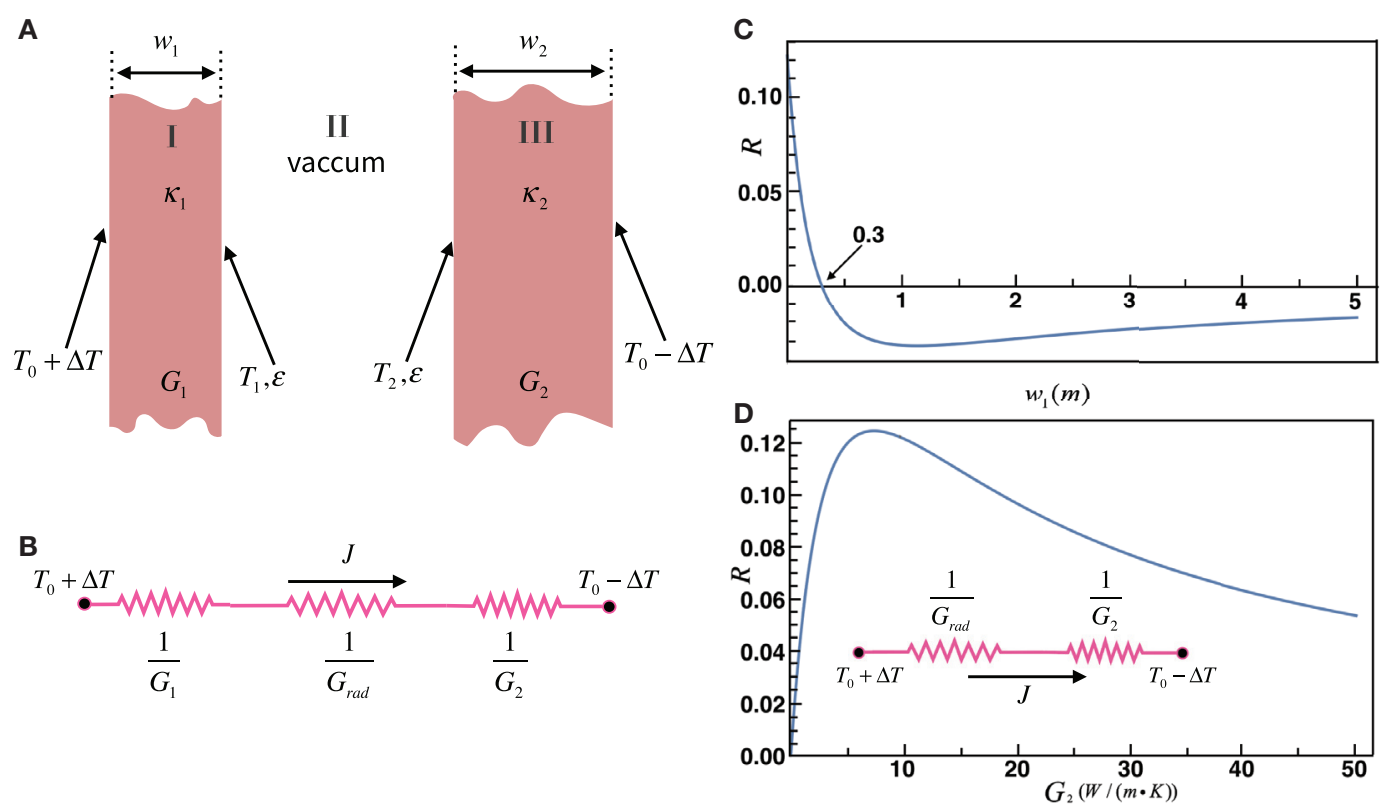

FIGURE 3 | (A) Sketch of the two infinite plate model. This is a one-dimensional model with three separated region. Temperature of two outer surfaces is set $T_{0}+\Delta T$ and $T_{0}-\Delta T$, respectively. $W_{1}$ and $W_{2}$ denotes the thickness of two infinite plate. $\varepsilon$ is the emissivity of the inner surface. $T_{1}$ and $T_{2}$ are measured as temperature of two inner surface. (B) Schematic graph depicting the equivalent thermal circuits of the plate-vaccum-plate sandwich model. Thermal resistance of conductance of region I and region III, and the equivalent thermal resistance of radiation in region II are in series. (C) $W_{1}$ versus rectification factor $R$. $T_{0}$ and $\Delta T$ are fixed at 318 and $25 \mathrm{~K}$. (D) $G_{2}$ versus rectification factor $R$ in the two-part model. $T_{0}$ and $\Delta T$ are fixed at 318 and $25 \mathrm{~K}$.

approximated as a homogenous material with conductance $G_{r a d}=\left.\frac{\partial\left(\varepsilon \sigma T^{4}\right)}{\partial T}\right|_{T=T_{0}}=4 \varepsilon \sigma T_{0}^{3}$, so that the equivalent conductance of the model is composed of thermal resistances of three regions in series: $G_{e}=\frac{1}{1 / G_{1}+1 / G_{2}+1 /\left(4 \varepsilon \sigma T_{0}^{3}\right)}$, with the temperature bias $2 \Delta T$. For a temperature difference $2 \Delta T$ smaller than $T_{0}$, the second order term is small compared with the first term. Therefore, the heat flux $J_{+}$and $J_{-}$is approximately proportional to the temperature difference $\Delta T$, which also explain the nearly linear relationship of $J_{+}$and $J_{-}$versus $\Delta T$ shown in the Figure 1C. Equation (2) also shows that the dominant first term of heat flux, although linear respect to thermal bias, has a nonlinear positive relationship with $T_{0}$, which well explains the nonlinear positive relationship and gradually saturated dependence on $T_{0}$, revealed in Figure 1D.

The second term in the heat flux expression equation (2) is very important to heat rectification. From the expression of heat 
flux, we can obtain the rectification factor up to the first order of thermal bias:

$$
R=\frac{J_{+}-J_{-}}{J-} \approx \frac{12 \varepsilon \sigma\left(G_{1}-G_{2}\right)}{G_{1} G_{2} / T_{0}^{3}+4 \varepsilon \sigma\left(G_{1}+G_{2}\right)} \frac{2 \Delta T}{T_{0}} .
$$

Therefore, the rectification coefficient is also linear to the $\Delta T$ as showed in Figure 1C and Figure 2C, which will vanish if the system becomes symmetric $G_{1}=G_{2}$. Through the analytical result, we can understand the nontrivial relationship between $R$ versus $T_{0}$, as shown in Figure 1D. Clearly from equation (2), when $T_{0}$ increases from low temperature, $R$ increases while when $T_{0}$ is already at high temperature, $R$ decreases as $T_{0}$ increases. With simple differential method, we can calculate that the denominator of the $R$ reaches its minimum value when $T_{0}=\left[\frac{G_{1} G_{2}}{2 \varepsilon \sigma\left(G_{1}+G_{2}\right)}\right]^{1 / 3}$, where $R$ has a maximum value when changing the average temperature $T_{0}$. This behavior coincides with what one observed in Figure 1D.

We also study the dependence of $R$ on the width $w_{1}$ in the plate-vacuum-plate sandwich model, to see the effect of competition between linear conduction and nonlinear radiation on the rectification. In Figure 3C, we keep $w_{2}$ fixed at $0.3 \mathrm{~m}$, and both $\kappa_{1}$ and $\kappa_{2}$ are equal to $1.7 \mathrm{~W} /(\mathrm{m} \cdot \mathrm{K})$. By changing $w_{1}$ only, we can see the importance of asymmetry in giving rise to the rectification that can be reversed. There is an exact vanishing point of $R$ at $w_{1}=w_{2}$, which means $G_{1}=G_{2}$ in this case. If we increase $w_{1}$ from the initial value $0.3 \mathrm{~m},|R|$ first increases with $w_{1}$, since the asymmetry of the system increases. However, while increasing $w_{1}$ further, the left conductance $G_{1}$ gets smaller, so the temperature difference at two inner surfaces $\left(T_{1}-T_{2}\right)$ gets smaller. Note that $T_{1}-T_{2}$ determines the flux (so as the total flux) through radiation within the nonlinear framework of Stefan-Boltzmann's Law, so when $w_{1}$ goes even larger, the radiative flux of nonlinear nature goes smaller so that the rectification $R$ falls down. When we decrease $w_{1}$ from the initial value $0.3 \mathrm{~m}, R$ increases quickly, and reaches a maximum at about $12 \%$. This is reasonable since when $w_{1}$ becomes smaller and smaller, approaching to $0, G_{1}$ becomes larger and gets close to infinity. The strongest asymmetry is achieved in this way, so that $R$ can reach its maximum limit in this case when $G_{1} \rightarrow \infty$.

To further maximize the rectification factor, we may consider a two-segment model with only region II and III where the left plate region $\mathrm{I}$ is removed. Temperature at the left boundary is imposed at the border of the vacuum part (region II). In Figure 3D, we show the numerical rectification factor $R$ vs $G_{2}$ with fixed $T_{0}$ and $\Delta T$ in the two-segment model. As we discussed above, the thermal rectification results from the competition between nonlinear radiation and linear conduction. When there is only one mechanism-either radiation or conduction, rectification can not appear since the system is symmetrical even with nonlinearity. Only if $G_{2}$ and $G_{\text {rad }}$ are comparable, which means the nonlinear and linear mechanisms are comparable to make the system most asymmetric, rectification effect becomes strongest. In other word, $R$ reaches its maximum around $G_{2} \approx G_{r a d}$. As shown in the case of Figure 3D, $G_{r a d}=4 \varepsilon \sigma T_{0}^{3}=7.3 \mathrm{~W} \mathrm{~m}^{-1} \mathrm{~K}^{-1}$, and $R$ indeed reaches the maximum when $G_{2}$ is about 7, comparable with $G_{\text {rad }}$.

\section{CONCLUSION AND DISCUSSION}

We have designed the asymmetric holey composites as a thermal rectifier, which has non-reciprocal heat transfer. The nonreciprocal heat transfer is attributed to the thermal radiation of nonlinear nature and the asymmetric geometric of the system. We have simulated and then confirmed from the theoretical analysis that the thermal rectification is approximately linear to the temperature difference of the system with the average temperature fixed and the maximal thermal rectification can be obtained by tuning the average temperature when the temperature difference is fixed. There have been considerable interests in realizing thermal rectifiers for its ability in thermal management and control for heat energy in the recent years. Most researches focus on achieving thermal rectifiers in nanoscale or even smaller, for its numerous potential applications in micro/nano-electronic cooling, thermal memory and computations. We have designed our thermal rectification model at macro-scale by inducing the nonlinearity with holes in the design, which is a simple model for thermal rectifiers. This means that the setup can be easily built, which might have potential applications in materials of architecture for diverse thermal management problems, e.g., energy-saving buildings, solar cells, and efficient refrigerator.

Furthermore, the rectification strength of the holely diodes can be further improved by constructing with two materials that with different temperature-dependent thermal conductivity of opposite trends [similar to the one in Dames (2009)]. In some way, the crucial nonlinearity induces the effective temperaturedependent conductance. For the nonlinear radiation, the conductance $G_{\text {rad }}=\frac{\varepsilon \sigma\left(T_{1}^{4}-T_{2}^{4}\right)}{T 1-T 2}=\varepsilon \sigma\left(T_{1}+T_{2}\right)\left(T_{1}^{2}+T_{2}^{2}\right)$, which under asymmetric geometry and bias reversing will effectively change the values. Therefore, a rectification action emerges. Beyond the nonlinear thermal radiation, the nonlinearity can also be introduced by the ballistic transport at quantum regime, since the phonon population is described by the Bose-Einstein distribution $N(\omega)=1 /\left[\exp \left(\hbar \omega / k_{B} T\right)-1\right]$ that has nonlinear dependence on temperature. Explicitly, the ballistic quantum thermal transport is described by the well-known LandauerBüttiker formula:

$$
J=\int_{0}^{\infty} d \omega \hbar \omega \mathcal{T}(\omega)\left[N_{L}(\omega)-N_{R}(\omega)\right],
$$

with $\mathcal{T}(\omega)$ the phononic transmission coefficient, $N_{L}(\omega)-N_{R}(\omega)$ the phonon population difference between two sides. When combining this ballistic transport with diffusive transport described by the linear Fourier's law and with asymmetric geometry of the system, the rectification emerges due to the nonlinear Bose-Einstein distribution. However, the rectification disappears in the high temperature classical regime since $1 /\left[\exp \left(\hbar \omega / k_{B} T\right)-1\right] \rightarrow k_{B} T / \hbar \omega$. As such, equation (4) reduces to the one with linear temperature dependence: $J=k_{B} \int_{0}^{\infty} d \omega \mathcal{T}(\omega)\left(T_{L}-T_{R}\right)$. Therefore, it is readily to prove that when combing with the Fourier's law, even with asymmetric geometry, the system can not have rectification. Both nonlinearity and asymmetry are necessary to construct the thermal rectifier (Li and Ren, 2014). 


\section{AUTHOR CONTRIBUTIONS}

GW and WZ carried out the simulation, performed the theoretical analysis, and contributed equally to this work. GW, WZ, HC, and JR, all contributed to discussion and the writing. JR conceived the project.

\section{REFERENCES}

Basu, S., and Francoeur, M. (2011). Near-field radiative transfer based thermal rectification using doped silicon. Appl. Phys. Lett. 98, 184301. doi:10.1063/1.3567026

Chotorlishvili, L., Etesami, S. R., Berakdar, J., Khomeriki, R., and Ren, J. (2015). Electromagnetically controlled multiferroic thermal diode. Phys. Rev. B 92, 134424. doi:10.1103/PhysRevB.92.134424

Dames, C. (2009). Solid-state thermal rectification with existing bulk materials. J. Heat Transfer 131, 177-181. doi:10.1115/1.3089552

Ding, H., Peng, G., Mo, S., Ma, D., Sharshir, S. W., and Yang, N. (2017). Ultra-fast vapor generation by a graphene nano-ratchet: a theoretical and simulation study. Nanoscale 9, 19066-19072. doi:10.1039/C7NR05304E

$\mathrm{Hu}$, B., and Yang, L. (2005). Heat conduction in the Frenkel-Kontorova model. Chaos 15, 1073. doi:10.1063/1.1862552

$\mathrm{Hu}$, S., An, M., Yang, N., and Li, B. (2017). A series circuit of thermal rectifiers: an effective way to enhance rectification ratio. Small 13, 1602726. doi:10.1002/ smll.201602726

Kobayashi, W., Teraoka, Y., and Terasaki, I. (2009). An oxide thermal rectifier. Appl. Phys. Lett. 95, 143501. doi:10.1063/1.3253712

Li, B., Wang, L., and Casati, G. (2004). Thermal diode: rectification of heat flux. Phys. Rev. Lett. 93, 184301. doi:10.1103/PhysRevLett.93.184301

Li, N., and Ren, J. (2014). Non-reciprocal geometric wave diode by engineering asymmetric shapes of nonlinear materials. Sci. Rep. 4, 6228-6228. doi:10.1038/ srep06228

Li, N., Ren, J., Wang, L., Zhang, G., Hanggi, P., and Li, B. (2012). Phononics: manipulating heat flow with electronic analogs and beyond. Rev. Mod. Phys. 84, 1045-1066. doi:10.1103/RevModPhys.84.1045

Li, S., Ding, X., Ren, J., Moya, X., Li, J., Sun, J., et al. (2014). Strain-controlled thermal conductivity in ferroic twinned films. Sci. Rep. 4, 6375. doi:10.1038/ srep06375

Maldovan, M. (2013). Sound and heat revolutions in phononics. Nature 503, 209-217. doi:10.1038/nature 12608

Otey, C. R., Lau, W. T., and Fan, S. (2010). Thermal rectification through vacuum. Phys. Rev. Lett. 104, 154301. doi:10.1103/PhysRevLett.104.154301

Ren, J. (2013). Predicted rectification and negative differential spin Seebeck effect at magnetic interfaces. Phys. Rev. B 88, 4045-4050. doi:10.1103/PhysRevB. 88.220406

Ren, J., Fransson, J., and Zhu, J.X. (2013). Nanoscale spin Seebeck rectifier: controlling thermal spin transport across insulating magnetic junctions with localized spin. Phys. Rev. B 89, 106-114. doi:10.1103/PhysRevB.89.214407

Ren, J., and Li, B. (2015). Phononics: controlling thermal energy, information carried by phonons and beyond. AIP Adv. 5, 294-303. doi:10.1063/ 1.4922168

Ren, J., and Zhu, J. X. (2013a). Heat diode effect and negative differential thermal conductance across nanoscale metal-dielectric interfaces. Phys. Rev. B Condens. Matter 87, 291-295. doi:10.1103/PhysRevB.87.241412

\section{FUNDING}

This work is supported by the National Natural Science Foundation of China (Grant Nos. 11775159 and 61621001), the National Key Research Program of China (No. 2016YFA0301101), and the National Youth 1000 Talents Program in China.

Ren, J., and Zhu, J. X. (2013b). Theory of asymmetric and negative differential magnon tunneling under temperature bias: towards a spin Seebeck diode and transistor Phys. Rev. B Condens. Matter 88, 3169-3182. doi:10.1103/PhysRevB.88.094427

Roberts, N. A., and Walker, D. G. (2011). A review of thermal rectification observations and models in solid materials. Int. J. Ther. Sci. 50, 648-662. doi:10.1016/j. ijthermalsci.2010.12.004

Ruokola, T., Ojanen, T., and Jauho, A. P. (2009). Thermal rectification in nonlinear quantum circuits. Phys. Rev. B Condens. Matter 79, 897-899. doi:10.1103/ PhysRevB.79.144306

Scheibner, R., Koenig, M., Reuter, D., Wieck, A. D., Buhmann, H., and Molenkamp, L. W. (2007). Quantum dot as thermal rectifier. New J. Phys. 10, 807-820. doi:10.1088/1367-2630/10/8/083016

Segal, D. (2008). Single mode heat rectifier: controlling energy flow between electronic conductors. Phys. Rev. Lett. 100, 105901. doi:10.1103/PhysRevLett.100.105901

Stevenson, P. F., Peterson, G. P., and Fletcher, L. S. (1991). Thermal rectification in similar and dissimilar metal contacts. J. Heat Transfer 113, 30-36. doi:10.1115/1.2910547

Terraneo, M., Peyrard, M., and Casati, G. (2002). Controlling the energy flow in nonlinear lattices: a model for a thermal rectifier. Phys. Rev. Lett. 88, 094302. doi:10.1103/PhysRevLett.88.094302

Wehmeyer, G., Yabuki, T., Monachon, C., Wu, J., and Dames, C. (2017). Thermal diodes, regulators, and switches: physical mechanisms and potential applications. Appl. Phys. Rev. 4, 041304. doi:10.1063/1.5001072

$\mathrm{Wu}, \mathrm{G}$., and Li, B. (2008). Thermal rectifier from deformed carbon nanohorns. J. Phys. Condens. Matter 20, 1871-1876. doi:10.1088/0953-8984/20/17/175211

Wu, L. A., and Segal, D. (2009). Sufficient conditions for thermal rectification in hybrid quantum structures. Phys. Rev. Lett. 102, 095503. doi:10.1103/ PhysRevLett.102.095503

Yang, N., Zhang, G., and Li, B. (2009). Thermal rectification in asymmetric graphene ribbons. Appl. Phys. Lett. 95, 033107. doi:10.1063/1.3183587

Zhao, X., Lee, Y. Y., and Liew, K. M. (2009). Mechanical and thermal buckling analysis of functionally graded plates. Compos. Struct. 90, 161-171. doi:10.1016/j. compstruct.2009.03.005

Conflict of Interest Statement: The authors declare that the research was conducted in the absence of any commercial or financial relationships that could be construed as a potential conflict of interest.

The reviewer, QC, and handling editor declared their shared affiliation.

Copyright (C) $2018 \mathrm{Zhu}, \mathrm{Wu}$, Chen and Ren. This is an open-access article distributed under the terms of the Creative Commons Attribution License (CC BY). The use, distribution or reproduction in other forums is permitted, provided the original author(s) and the copyright owner are credited and that the original publication in this journal is cited, in accordance with accepted academic practice. No use, distribution or reproduction is permitted which does not comply with these terms. 